The authors concluded that higher rates of vancomycin or third-generation cephalosporin use were associated with increased prevalence of VRE, independent of other ICU characteristics and the endemic VRE prevalence elsewhere in the hospital. Decreasing the use rates of these antimicrobial agents could reduce rates of VRE in ICUs.

FROM: Fridkin SK, Edwards JR, Courval JM, Hill H, Tenover FC, Lawton $\mathrm{R}$, et al. The effect of vancomycin and third-generation cephalosporins on prevalence of vancomycin-resistant enterococci in 126 U.S. adult intensive care units. Ann Intern Med 2001;135:175-183.

\section{Risk Factors for Ventilator-Associated Pneumonia in a Community Hospital}

Ibrahim and coinvestigators from Washington University School of Medicine, Barnes-Jewish Hospital, St Louis, Missouri, conducted a study to identify prospectively the occurrence of ventilator-associated pneumonia (VAP) in a community hospital and to determine the risk factors for VAP and the influence of VAP on patient outcomes in a nonteaching institution. The study was a prospective cohort study conducted in a medical ICU and a surgical ICU in a 500-bed private community nonteaching hospital. All patients receiving mechanical ventilation who were admitted to the ICU setting between March 1998 and December 1999 were prospectively evaluated.

During a 22-month period, 3,171 patients were admitted to the medical and surgical ICUs. Eight hundred eighty patients $(27.8 \%)$ received mechanical ventilation. VAP developed in 132 patients (15.0\%) receiving mechanical ventilation. Three hundred one patients (34.2\%) who received mechanical ventilation died during hospitalization. Logistic-regression analysis demonstrated that tracheostomy (adjusted odds ratio [AOR], 6.71; 95\% confidence interval $\left.\left[\mathrm{CI}_{95}\right], 3.91-11.50 ; P<.001\right)$, multiple central venous-line insertions ( $\mathrm{AOR}, 4.20 ; \mathrm{CI}_{95}, 2.72-6.48 ; P<.001$ ), reintubation (AOR, 2.88; $\mathrm{CI}_{95}, 1.78-4.66 ; P<.001$ ), and the use of antacids (AOR, 2.81; $\mathrm{CI}_{95}, 1.19-6.64 ; P=.019$ ) were independently associated with the development of VAP. The hospital mortality of patients with VAP was significantly greater than the mortality of patients without VAP ( $45.5 \%$ vs $32.2 \%$, respectively; $P=.004$ ). The occurrence of bacteremia, compromised immune system, higher APACHE II scores, and older age were identified as independent predictors of hospital mortality.

The authors concluded that VAP is a common nosocomial infection in the community hospital setting. The risk factors for the development of VAP and risk factors for hospital mortality in a community hospital are similar to those identified from university-affiliated hospitals. These risk factors can potentially be employed to develop local strategies for the prevention of VAP. ICU clinicians should be aware of the risk factors associated with the development of VAP and the impact of VAP on clinical outcomes. More importantly, they should cooperate in the development of local multidisciplinary strategies aimed at the prevention of VAP and other nosocomial infections.
FROM: Ibrahim EH, Tracy L, Hill C, Fraser VJ, Kollef $\mathrm{MH}$. The occurrence of ventilator-associated pneumonia in a community hospital: risk factors and clinical outcomes. Chest 2001;120:555-561.

\section{Touch Contamination Levels During Anesthetic Procedures: Relation to Hand- Hygiene Procedures}

Merry and colleagues from the University of Auckland, New Zealand, have reported on a study of microbial contamination levels during anesthetic procedures. After different methods of hand preparation, volunteers rolled segments of a sterile central venous catheter between their fingertips, and bacterial transfer was evaluated by standardized quantitative culture. The number of bacteria transferred differed between methods $(P<.001)$. Comparisons were made with the control group (no preparation at all; median, third quartile and maximum count $=6.5,24,55)$. Bacterial transfer was greatly increased with wet hands $(1,227,1,932,3,254 ; P<.001)$. It was reduced with a new rapid method, based on thorough drying with a combination of 10 seconds using a cloth towel followed by either 10 or 20 seconds with a hot-air towel $(0,3,7$ and 0 , 4,30 , respectively; $P=.007$ and .004 , respectively).

When asked to follow their personal routines, 10 consultant anesthetists used a range of methods. Collectively, these were not significantly better than control $(7.5,15,55$; $P=.73)$ and neither was an air towel alone $(2.5,15,80 ; P=.176)$ nor the hospital's standard procedure $(0,1,500 ; P=.035)$.

The authors concluded that, if hand preparation is needed, an adequate and validated method should be used, together with thorough hand drying.

FROM: Merry AF, Miller TE, Findon G, Webster CS, Neff SP. Touch contamination levels during anaesthetic procedures and their relationship to hand hygiene procedures: a clinical audit. Br J Anaesth 2001;87:291-294.

\section{Evolution of MRSA}

Crisostomo and colleagues have theorized that the key genetic component of methicillin resistance, the mecA determinant, is not native to Staphylococcus aureus. Thus, the evolution of methicillin-resistant $S$ aureus (MRSA) must have begun with the acquisition of the mecA determinant from an unknown heterologous source some time before the first reported appearance of MRSA isolates in clinical specimens in the United Kingdom and Denmark (in the early 1960s). They compared the genetic backgrounds and phenotypes of a group of methicillin-susceptible $S$ aureus (MSSA) isolates to the properties of MRSA strains isolated in Denmark and the United Kingdom during the same time period and to the genetic profiles of contemporary epidemic clones of MRSA.

All early MRSA isolates resembled a large group of the early MSSA blood isolates in phenotypic and genetic properties, including phage group, antibiotype (resistance to penicillin, streptomycin, and tetracycline), pulsed-field gel electrophoresis pattern, and spaA type and multilocus 
sequence type, strongly suggesting that the early MSSA examined here represented the progeny of a strain that served as one of the first $S$ aureus recipients of the methicillin-resistance determinant in Europe. The genetic background of this group of early MSSA isolates was also very similar to that of the widely disseminated contemporary "Iberian clone" of MRSA, suggesting that genetic determinants present in early MSSA and essential for some aspects of the epidemicity or virulence of these strains, may have been retained by this highly successful contemporary MRSA lineage.

FROM: Crisostomo MI, Westh H, Tomasz A, Chung M, Oliveira DC, de Lencastre. The evolution of methicillin resistance in Staphylococcus aureus: similarity of genetic backgrounds in historically early methicillin-susceptible and -resistant isolates and contemporary epidemic clones. Proc Natl Acad Sci U S A 2001;98:9865-9870.

\section{Detection of Nosocomial Tuberculosis With Molecular Typing}

Tudo and coinvestigators from Barcelona, Spain, conducted a study to investigate the use of restriction fragment-length polymorphism to detect unsuspected cases of nosocomial transmission of TB among patients who had been admitted to a university hospital. One hundred fifty-one samples of Mycobacterium tuberculosis isolated from patients with pulmonary TB were studied. The isolates from 37 patients (24.5\%) defined 11 clusters. None of the patients infected with these cluster isolates had hospital stays that coincided with one another, and, for $5.4 \%$ of the patients, the epidemiological link clearly was outside the hospital. Previous incarceration was associated with infection with cluster isolates. In addition, 109 patients without TB (41 of whom were infected with HIV) who shared a room with patients who had TB were followed for 18 to 60 months. Among the patients who survived, secondary cases of TB due to nosocomial transmission were not detected.

FROM: Tudo G, Gonzalez J, Gatell JM, Cayla JA, Martinez E, Garcia A, et al. Detection of unsuspected cases of nosocomial transmission of tuberculosis by use of a molecular typing method. Clin Infect Dis 2001;33:453-459.

\section{Nasal Carriage of $S$ aureus: Risk Factor for Skin Infections}

Toshkova and coinvestigators have reported on a study designed to investigate the significance and the relation between nasal carriage of Staphylococcus aureus and staphylococcal skin infections. Thirty-one $S$ aureus strains, isolated from 12 patients with chronic and recurrent skin infections, one patient with septicemia, and one patient with otitis externa were studied. The staphylococcal strains were isolated from the site of infection and from the anteri- or nares of each patient. The identity of both strains of each pair could be demonstrated by determination of phenotypic properties and by genotyping of the isolates. The phenotypic properties included hemolytic activities, antibiotic resistance data, and the production of enterotoxins. The identity was additionally confirmed by phage typing, by determination of the size and the number of repeats of the X region of $s p a$ gene, by determination of gene polymorphisms of coa gene, and by macrorestriction analysis of the chromosomal DNA of the isolates by pulsed-field gel electrophoresis.

The results showed an identity of the $S$ aureus obtained from anterior nares and from skin infection of each patient, indicating the importance of nasal carriage of these bacteria for development of human skin infection.

FROM: Toshkova $\mathrm{K}$, Annemuller $\mathrm{C}$, Akineden $O$, Lammler $C$. The significance of nasal carriage of Staphylococcus aureus as risk factor for human skin infections. FEMS Microbiol Lett 2001;202:17-24.

\section{Attitudes Toward Infection Control Programs in Australia}

Debate remains over the core activities of infection control (IC) programs. Differences in stakeholder opinions must be considered if consensus panel guidelines and recommendations are to be applied broadly. Murphy and McLaws have reported on a survey of administrators and clinicians employed in hospitals in New South Wales, Australia. Respondents self-reported their levels of agreement with affirmative statements regarding the role of the infection control practitioner (ICP) and the essential requirements and infrastructure of IC programs. The study population included administrators and clinicians in each public, private, and freestanding day hospital in New South Wales. Respondents reported the intensity of their agreement with 16 affirmative statements relating to IC program infrastructure and resources and the ICP's role and responsibilities.

The overall response rate was $62.1 \%$ (587/945). Clinicians $(349 / 587)$ and administrators (238/587) accounted for $59.5 \%$ and $40.5 \%$ of the response rate, respectively. Overall, administrators and clinicians reported greatest levels of agreement for those elements not requiring additional resources.

The authors concluded that the extent of divergence between administrators and clinicians is not so great that it cannot be resolved. They advocate better communication between clinicians and administrators in conjunction with objective strategic planning and offer their findings as a guide for ICPs to either establish or negotiate the core components of their IC program.

FROM: Murphy CL, McLaws Ml. Variation in administrators' and clinicians' attitudes toward critical elements of an infection control program and the role of the infection control practitioner in New South Wales, Australia. Am J Infect Control 2001;29:262-270. 